\title{
ESTIMATION OF SCALE PARAMETER OF MORGENSTERN TYPE BIVARIATE GENERALIZED UNIFORM DISTRIBUTION BY RANKED SET SAMPLING
}

\author{
Kirtee K. Kamalja and Rohan D. Koshti \\ Department of Statistics, School of Mathematical Sciences, \\ Kavayitri Bahinabai Chaudhari North Maharashtra University, Jalgaon, India \\ kirteekamalja@gmail.com,rohankoshti5@yahoo.co.in
}

\begin{abstract}
The concept of ranked set sampling (RSS) is applicable whenever ranking on a set of sampling units can be done easily by a judgment method or based on an auxiliary variable. In this work, we consider a study variable $Y$ correlated with auxiliary variable $X$ which is used to rank the sampling units. Further $(X, Y)$ is assumed to have Morgenstern type bivariate generalized uniform distribution. We obtain an unbiased estimator of a scale parameter associated with the study variable $Y$ based on different RSS schemes and censored RSS. Efficiency comparison study of these estimators is also performed and presented numerically.
\end{abstract}

Key words: Ranked set sampling, concomitants of order statistics, Morgenstern type bivariate generalized uniform distribution, best linear unbiased estimator. 


\section{Introduction}

The concept of ranked set sampling (RSS) was first introduced by McIntyre (1952) for improving the precision of the sample mean, an estimator of population mean and applied to the problem of estimating the mean pasture yields. This concept is applicable whenever variable of interest is difficult or expensive to measure but ranking on a small set of measurement is easily available. For an overview see, Patil, Sinha, and Taillie (1994), Barnett (1999), Chen, Bai, and Sinha (2004), Wolfe (2004). McIntyre (1952) uses judgement method for ranking a set of sample units. Judgement method is not suitable when there is ambiguity in discriminating the rank of one unit with another. Also, the imperfect ranking of the units in RSS leads to larger mean square errors of RSS estimators. To remedy this problem Stokes (1977) uses an auxiliary variable for ranking of the sampling units. Stokes (1977) considers a situation where the variable of interest, say $Y$, is difficult or expensive to measure, but an auxiliary variable $X$ correlated with $Y$ is easily measurable and can be ordered exactly. The procedure of RSS using auxiliary variable described by Stokes (1977) is as follows.

Choose $n$ independent bivariate samples each of size $n$ and observe the value of the auxiliary variable $X$ on each of these units. For the first sample, select that unit for which the measurement on the auxiliary variable $X$ is the smallest and measure the $Y$ variate associated with it. In the second sample, choose $Y$ associated to the second smallest $X$. This procedure is repeated until $Y$ associated with the largest $X$ in the last sample is measured. The resulting set of $n$ units as described above is called RSS. Let $\left(X_{(r) r}, Y_{[r] r}\right), r=1,2, \ldots, n$ be the pair selected from the $r^{\text {th }}$ pair of sample, where $X_{(r) r}$ denote the $r^{\text {th }}$ order statistic of the auxiliary variable in the $r^{\text {th }}$ sample and $Y_{[r] r}$ denote the measurement made on the $Y$ variate associated with $X_{(r) r}$. David and Nagaraja (2003) referred $Y_{[r] r}$ as the concomitant of the $r^{\text {th }}$ order statistic arising from the $r^{\text {th }}$ sample.

RSS procedure described by Stokes (1977) has found many diverse applications in environmental, agricultural and ecological studies and used effectively to estimate the parameters associated with the environmental variables. Bain (1978) discusses a striking example of RSS where a study variate $Y$ represents the oil pollution of sea water and the auxiliary variable $X$ represents the tar deposit in the nearby sea shore. Clearly collecting sea water sample and measuring the oil pollution in it is strenuous and expensive. However, the prevalence of pollution in the sea water is much reflected by the tar deposit in the surrounding terminal sea shore. In this example ranking the pollution level of sea water based on the tar deposit on the sea shore is more natural and scientific than ranking it visually or by judgement method.

Stokes (1977) proposes RSS mean as an estimator for the mean of the study variate $Y$, when an auxiliary variable $X$ is used for ranking the sample units, under the assumption that $(X, Y)$ follow a bivariate Normal distribution. Further Barnett and Moore (1997) obtained the best linear unbiased estimator (BLUE) of the mean of the study variate $Y$, based on a RSS for the same situation. Stokes (1980) introduces a modified RSS procedure in which only the largest or the smallest judgment ranked unit is chosen for quantification. Stokes (1995) studies the estimation of parameters in location-scale family distribution using RSS. Samawi, Ahmed, and Abu-Dayyeh (1996) investigated the use of a variety of extreme RSS (ERSS) for estimating the population mean. Lam, Sinha, and Wu (1994) used RSS to estimate the parameters of a two-parameter exponential distribution. Further Lam, Sinha, and Wu (1996) estimated the location and scale parameters of a logistic distribution using RSS. 
Another scheme of RSS was investigated by Al-Odat and Al-Saleh (2001) which is moving extreme RSS (MERSS). Shaibu and Muttalak (2004) estimated the parameters of the Normal, Exponential and Gamma distribution using Median RSS and ERSS. Al-Saleh and Al-Ananbeh (2007) proposed the concept of MERSS with concomitant variable for the estimation of mean of the bivariate normal distribution. Chacko and Thomos (2007) obtained the BLUE of the parameter involved in the study variate $Y$, based on a RSS for bivariate Pareto distribution. Chacko and Thomos (2008) estimated the parameters of Morgenstern type bivariate exponential distribution by RSS and censored RSS scheme. Tahmasebi and Jafari (2012) obtained several estimators for a scale parameter of Morgenstern type bivariate uniform distribution by RSS, Upper ranked set sample (URSS), ERSS and MERSS. Lesitha and Thomas (2013) obtained an estimator of scale parameter of Morgenstern type bivariate log-logistic distribution by RSS and ERSS. Hanandeh and Al-Saleh (2013) estimated the parameters of Downton's bivariate exponential distribution using MERSS. Tahmasebi and Jafari (2014) considered the Morgenstern type bivariate generalized exponential distribution and obtained several estimators for the population mean. Singh and Mehta (2015) obtained an estimator of scale parameter of a Morgenstern type bivariate uniform distribution by RSS and censored RSS scheme. Chacko (2016) investigated a new RSS scheme called ordered extreme RSS and obtained an estimator of the parameter for Morgenstern type bivariate exponential distribution. Koshti and Kamalja (2017) obtained an estimator of scale parameter by RSS and censored RSS for bivariate Lomax distribution.

The above review of the literature shows the importance of RSS schemes in estimation of parameters for different bivariate distributions. In this paper, we consider a Morgenstern type bivariate generalized uniform (MTBGU) distribution and use different RSS and censored RSS schemes to estimate the scale parameter associated with study variable. The present work is organized as follows.

In section 2, we discuss the general theory of Morgenstern type bivariate generalized (MTBG) distribution and briefly review concomitants of order statistics (COS) for MTBGU distribution obtained by Scaria and Thomas (2008). In section 3, we obtain an unbiased estimator of scale parameter of MTBGU distribution based on RSS, LRSS, URSS, ERSS and MERSS schemes. Further in section 4, we obtain censored RSS estimator of scale parameter of MTBGU distribution. We perform an efficiency comparison of estimators obtained in section 3 numerically and present them graphically in section 5. Section 6, concludes the paper with final remarks.

\section{Concomitants of Order Statistics for Morgenstern type bivariate generalized uniform distribution}

In modelling the bivariate data, when the prior information is in the form of marginal distributions, it is of advantage to consider families of bivariate distributions with specified marginals. Morgenstern (1956) provides a flexible family in such context. One important limitation of Morgenstern family is that its correlation coefficient is restricted to a narrow range $\left(-\frac{1}{3}, \frac{1}{3}\right)$. Accordingly, distributions belonging to Morgenstern family can be used to model the data that exhibits low correlation. In order to enhance the range of correlation between the variables, several modifications to the Morgenstern family have been suggested in the literature. Several researchers like Cambanis (1977), Huang and Kotz (1984, 1999), Bairamov, Kotz, and Bekci (2001), Bairamov and Kotz (2002), Veena and Thomas (2008) extended Morgenstern family to enhance the range of correlation between variables and to 
impart more flexibility to a new family. Veena and Thomas (2008) introduced a family of distributions which constitutes a natural generalization of the bivariate Morgenstern family.

The distribution function of Morgenstern type bivariate generalized (MTBG) distribution for a bivariate random variable $(X, Y)$ given by Veena and Thomas (2008) is,

$$
F(x, y)=F_{X}(x) F_{Y}(y)+\sum_{i=1}^{t} \alpha_{i}\left\{F_{X}(x)\left[1-F_{X}(x)\right]\right\}^{m_{i}}\left\{F_{Y}(y)\left[1-F_{Y}(y)\right]\right\}^{p_{i}}
$$

where $F_{X}(x)$ and $F_{Y}(y)$ are the marginals of $F(x, y), m_{i}, p_{i}$ are real constants $\geq 1, \alpha_{i}$ is a real constant constrained to lie in an interval about zero and $t$ is a positive integer. Scaria and Thomas (2008) considered the distribution with $t=1$ as follows.

$$
F(x, y)=F_{X}(x) F_{Y}(y)+\alpha\left\{F_{X}(x)\left[1-F_{X}(x)\right]\right\}^{m}\left\{F_{Y}(y)\left[1-F_{Y}(y)\right]\right\}^{p}
$$

For uniform marginals, the distribution function of MTBG (i.e. MTBGU) distribution is given by,

$$
F(x, y)=\frac{x}{\theta_{1}} \frac{y}{\theta_{2}}+\alpha\left[\frac{x}{\theta_{1}}\left(1-\frac{x}{\theta_{1}}\right)\right]^{m}\left[\frac{y}{\theta_{2}}\left(1-\frac{y}{\theta_{2}}\right)\right]^{p} ; 0<x<\theta_{1}, 0<y<\theta_{2}
$$

It is denoted by $(X, Y) \sim \operatorname{MTB} G U\left(\theta_{1}, \theta_{2}, m, p, \alpha\right)$ distribution where $\theta_{1}, \theta_{2}$ are scale parameters, $m, p$ are shape parameters and $\alpha$ is the association parameter.

The MTBGU distribution with $m=1$ and $p=1$ reduces to Morgenstern type bivariate uniform (MTBU) distribution. Each marginal of the above MTBGU distribution is univariate uniform. Particularly, marginal distribution of $Y$ is $U\left(0, \theta_{2}\right)$.

Scaria and Nair (1999) studied the COS for MTBU distribution. (i.e. $\left.\operatorname{MTBGU}\left(\theta_{1}, \theta_{2}, 1,1, \alpha\right)\right)$ where as Scaria and Thomas (2008) obtained the COS for MTBGU distribution. The $p d f$ of concomitants of the $r^{t h}$ order statistic $Y_{[r] n}$ is given by,

$$
f_{Y_{[r] n}}(y)=\frac{1}{\theta_{2}}\left[1+\alpha m p \frac{B(m+r-1, m+n-r)}{B(r, n-r+1)} \frac{n-2 r+1}{2 m+n-1}\left(\frac{y}{\theta_{2}}\left(1-\frac{y}{\theta_{2}}\right)\right)^{p-1}\left(1-\frac{2 y}{\theta_{2}}\right)\right] ; 0<y<\theta_{2}
$$

where $B(.,$.$) denotes beta function.$

The mean and variance of $Y_{[r] n}$ is given by,

$$
E\left(Y_{[r] n}\right)=\theta_{2} \xi_{r} \quad \text { and } \quad \operatorname{Var}\left(Y_{[r] n}\right)=\theta_{2}^{2} \delta_{r}
$$

where $\xi_{r}=\frac{1}{2}-\alpha m p \frac{B(m+r-1, m+n-r)}{B(r, n-r+1)} \frac{n-2 r+1}{2 m+n-1} \frac{B(p, p+1)}{2 p+1}$ and

$$
\delta_{r}=\frac{1}{12}-\left(\alpha m p \frac{B(m+r-1, m+n-r)}{B(r, n-r+1)} \frac{n-2 r+1}{2 m+n-1} \frac{B(p, p+1)}{2 p+1}\right)^{2} .
$$

We observe that the constants $\xi_{r}$ and $\delta_{r}$ satisfy the following for different values of $m$ and $p$.

i) $\quad \xi_{r}+\xi_{n-r+1}=1, r=1,2, \ldots, n$ and consequently $\sum_{r=1}^{n} \xi_{r}=n / 2$.

ii) $\delta_{r}=\delta_{n-r+1}, r=1,2, \ldots, n$

iii) $\delta_{r}>0$ and $\sum_{r=1}^{n} \delta_{r} \leq n / 12$

iv) $\xi_{r}>0$ and $\sum_{r=1}^{n} \xi_{r}^{2} / \delta_{r} \geq 3 n$ 


\section{Estimator of scale parameter $\boldsymbol{\theta}_{2}$ based on different RSS schemes}

In this section, we obtain an unbiased estimator of the scale parameter $\theta_{2}$ based on different RSS schemes when $m, p$ and $\alpha$ are known. Further we compare different estimators of $\theta_{2}$ obtained by using different RSS schemes.

\subsection{RSS estimation}

Suppose $n$ random samples each of size $n$ are drawn from $\operatorname{MTBGU}\left(\theta_{1}, \theta_{2}, m, p, \alpha\right)$ distribution. Let $X_{(r) r}$ be the $r^{\text {th }}$ order statistic of the auxiliary variable $X$ in the $r^{\text {th }}$ sample and $Y_{[r] r}$ be the measurement on the $Y$ variate associated with $X_{(r) r}, r=1,2, \ldots, n$. Here $Y_{[r] r}$ is referred as the concomitant of $r^{\text {th }}$ order statistic and $Y_{[1] 1}, Y_{[2] 2}, \ldots, Y_{[n] n}$ is referred to as RSS from MTBGU distribution. The handling of these RSS observations for inference problem will be easy since observations are uncorrelated as they are drawn from independent samples.

Based on $\operatorname{RSS} Y_{[1] 1}, Y_{[2] 2}, \ldots, Y_{[n] n}$ from $M T B G U$ distribution, we obtain an unbiased estimator of a scale parameter $\theta_{2}$ under the assumption that $m, p$ and $\alpha$ are known. The estimator $\hat{\theta}_{2, R S S}$ is defined as,

$$
\widehat{\theta}_{2, R S S}=\frac{2}{n} \sum_{r=1}^{n} Y_{[r] n}
$$

Clearly $\hat{\theta}_{2, R S S}$ is the unbiased estimator of $\theta_{2}$ and its variance is given by,

$$
\operatorname{Var}\left(\hat{\theta}_{2, R S S}\right)=\frac{4 \theta_{2}^{2}}{n^{2}} \sum_{r=1}^{n} \delta_{r}
$$

The unbiased estimator of $\theta_{2}$ based on a simple random sample (SRS) $y_{1}, y_{2}, \ldots, y_{n}$ from $U\left(0, \theta_{2}\right)$ distribution is $\hat{\theta}_{2, S R S}=2 \bar{y}$ where $\bar{y}=\frac{1}{n} \sum_{r=1}^{n} y_{r}$ and $\operatorname{Var}\left(\hat{\theta}_{2, S R S}\right)=\theta_{2}^{2} / 3 n$. The efficiency of $\hat{\theta}_{2, R S S}$ over $\hat{\theta}_{2, S R S}$ is,

$$
e_{1}=R E\left(\hat{\theta}_{2, R S S} \mid \hat{\theta}_{2, S R S}\right)=\frac{n}{12 \sum_{r=1}^{n} \delta_{r}} .
$$

Observe that $e_{1} \geq 1$, since $\sum_{r=1}^{n} \delta_{r} \leq n / 12$. Hence $\hat{\theta}_{2, R S S}$ is more efficient than $\hat{\theta}_{2, S R S}$.

\subsection{BLUE using RSS}

We obtain BLUE of $\theta_{2}$ based on RSS under the assumption that $m, p$ and $\alpha$ are known.

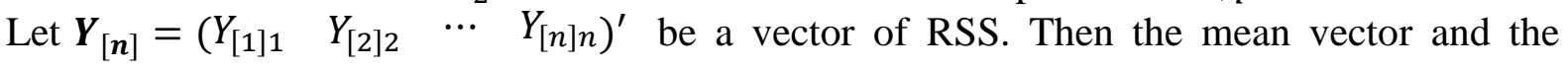
dispersion matrix of $\boldsymbol{Y}_{[\boldsymbol{n}]}$ is given by,

$$
\begin{aligned}
& E\left(\boldsymbol{Y}_{[\boldsymbol{n}]}\right)=\theta_{2} \xi, \\
& D\left(\boldsymbol{Y}_{[\boldsymbol{n}]}\right)=\theta_{2}^{2} G .
\end{aligned}
$$

where $\xi=\left(\begin{array}{llll}\xi_{1} & \xi_{2} & \cdots & \xi_{n}\end{array}\right)^{\prime}$

and $\quad G=\operatorname{diag}\left(\delta_{1}, \delta_{2}, \ldots, \delta_{n}\right)$. 
The BLUE $\hat{\theta}_{2, B L U E}$ of $\theta_{2}$ based on RSS using the generalized linear model for $E\left(\boldsymbol{Y}_{[\boldsymbol{n}]}\right)=\theta_{2} \xi$ with $D\left(\boldsymbol{Y}_{[\boldsymbol{n}]}\right)=\theta_{2}^{2} G$ (David and Nagaraja (2003)) is given by,

$$
\widehat{\theta}_{2, B L U E}=\left(\xi^{\prime} G^{-1} \xi\right)^{-1} \xi^{\prime} G^{-1} \boldsymbol{Y}_{[n]} .
$$

The variance of $\hat{\theta}_{2, B L U E}$ is given by,

$$
\operatorname{Var}\left(\hat{\theta}_{2, B L U E}\right)=\left(\xi^{\prime} G^{-1} \xi\right)^{-1} \theta_{2}^{2}
$$

Simplifying (1) and (2) we get,

$$
\hat{\theta}_{2, B L U E}=\frac{\sum_{r=1}^{n}\left(\xi_{r} / \delta_{r}\right) Y_{[r] n}}{\sum_{r=1}^{n} \xi_{r}^{2} / \delta_{r}}, \quad \quad \operatorname{Var}\left(\hat{\theta}_{2, B L U E}\right)=\frac{\theta_{2}^{2}}{\sum_{r=1}^{n} \xi_{r}^{2} / \delta_{r}} .
$$

The efficiency of $\hat{\theta}_{2, B L U E}$ over $\hat{\theta}_{2, R S S}$ is,

$$
e_{2}=R E\left(\hat{\theta}_{2, B L U E} \mid \hat{\theta}_{2, R S S}\right)=\frac{4}{n^{2}}\left(\sum_{r=1}^{n} \delta_{r}\right)\left(\sum_{r=1}^{n} \frac{\xi_{r}^{2}}{\delta_{r}}\right)
$$

It is observed that $\sum_{r=1}^{n} \frac{\xi_{r}^{2}}{\delta_{r}} \geq 3 n$. Hence $\hat{\theta}_{2, B L U E}$ is more efficient than $\hat{\theta}_{2, R S S}$ as $\sum_{r=1}^{n} \delta_{r} \leq \frac{n}{12}$.

\subsection{LRSS and URSS estimation}

Stokes (1980) introduces a modified RSS procedure in which only the largest or the smallest judgment ranked unit is chosen for quantification. In lower (upper) RSS, measurement on $Y$ is made on those units for which measurement on $X$ is minimum (maximum). This is done for each of the $n$ samples. The sample observations $Y_{[1] 1}, Y_{[1] 2}, \ldots, Y_{[1] n}\left(Y_{[n] 1}, Y_{[n] 2}, \ldots, Y_{[n] n}\right)$ is referred as LRSS (URSS). The BLUE $\hat{\theta}_{2, L R S S}$ and $\hat{\theta}_{2, U R S S}$ of $\theta_{2}$ based on LRSS and URSS are as follows.

$$
\hat{\theta}_{2, L R S S}=\frac{1}{n \xi_{1}} \sum_{r=1}^{n} Y_{[1] r}, \quad \hat{\theta}_{2, U R S S}=\frac{1}{n \xi_{n}} \sum_{r=1}^{n} Y_{[n] r},
$$

The variances of these estimators are,

$$
\operatorname{Var}\left(\hat{\theta}_{2, L R S S}\right)=\frac{\theta_{2}^{2} \delta_{1}}{n \xi_{1}^{2}}, \quad \operatorname{Var}\left(\hat{\theta}_{2, U R S S}\right)=\frac{\theta_{2}^{2} \delta_{n}}{n \xi_{n}^{2}}
$$

where $\delta_{1}=\delta_{n}=\frac{1}{12}-\left(\alpha m p \frac{B(m+n-1, m)}{B(1, n)} \frac{n-1}{2 m+n-1} \frac{B(p, p+1)}{2 p+1}\right)^{2}$,

$\xi_{1}=\frac{1}{2}-\alpha m p \frac{B(m+n-1, m)}{B(1, n)} \frac{n-1}{2 m+n-1} \frac{B(p, p+1)}{2 p+1}$ and $\xi_{n}=\frac{1}{2}-\alpha m p \frac{B(m+n-1, m)}{B(1, n)} \frac{1-n}{2 m+n-1} \frac{B(p, p+1)}{2 p+1}$.

The efficiency of $\hat{\theta}_{2, L R S S}$ and $\hat{\theta}_{2, U R S S}$ over $\hat{\theta}_{2, R S S}$ and $\hat{\theta}_{2, B L U E}$ is given by,

$$
e_{3}=R E\left(\hat{\theta}_{2, L R S S} \mid \hat{\theta}_{2, R S S}\right)=\frac{4 \xi_{1}^{2}\left(\sum_{r=1}^{n} \delta_{r}\right)}{n \delta_{1}}, \quad e_{4}=R E\left(\hat{\theta}_{2, L R S S} \mid \hat{\theta}_{2, B L U E}\right)=\frac{n \xi_{1}^{2} / \delta_{1}}{\sum_{r=1}^{n} \xi_{r}^{2} / \delta_{r}}
$$




$$
e_{5}=R E\left(\hat{\theta}_{2, U R S S} \mid \hat{\theta}_{2, R S S}\right)=\frac{4 \xi_{n}^{2}\left(\sum_{r=1}^{n} \delta_{r}\right)}{n \delta_{n}}, \quad e_{6}=R E\left(\hat{\theta}_{2, U R S S} \mid \hat{\theta}_{2, B L U E}\right)=\frac{n \xi_{n}^{2} / \delta_{n}}{\sum_{r=1}^{n} \xi_{r}^{2} / \delta_{r}}
$$

Observe that as $\left.\xi_{1}^{2}\right|_{\alpha_{3}}=\left.\xi_{n}^{2}\right|_{-\alpha_{3}}$, therefore $\left.e_{3}\right|_{\alpha_{3}}=\left.e_{5}\right|_{-\alpha_{3}}$ and $\left.e_{4}\right|_{\alpha_{3}}=\left.e_{6}\right|_{-\alpha_{3}}$.

\subsection{ERSS estimation}

Samawi, Ahmed, and Abu-Dayyeh (1996) introduced ERSS which is easier than usual RSS procedure. Based on the even or odd sample size, ERSS can be chosen in two ways. For both procedures, the selection of first $n-1$ units are same, but the selection of $n^{\text {th }}$ unit depends on whether the sample size is even or odd. The procedure is as follows.

The ERSS scheme involves randomly choosing $n$ samples each of $n$ units from the population. From the first set of $n$ units, select the smallest $X$ and measure the $Y$ variate associated with the smallest $X$ variate. From the second set of $n$ units, select the largest $X$ and measure the $Y$ variate associated with the largest $X$ variate. From the third set of $n$ units, select the smallest $X$ and measure the $Y$ variate associated with the smallest $X$ variate and so on. This is repeated until we obtain the first $n-1$ units.

The choice of the $n^{\text {th }}$ unit from the $n^{\text {th }}$ set depends on whether $n$ is even or odd. If $n$ is even, select the largest $X$ and measure the $Y$ variate associated with the largest $X$ variate. Such a sample will be denoted by $\mathrm{ERSS}_{1}$ and is $\left(Y_{[1] 1}, Y_{[n] 2}, \ldots, Y_{[1] n-1}, Y_{[n] n}\right)$.

If $n$ is odd, the $n^{\text {th }}$ unit can be chosen in two different ways.

a) For the measure of the $n^{\text {th }}$ unit, the average of $Y$ variates associated with the smallest and the largest $X$ in the $n^{\text {th }}$ set is chosen. In this case the selected sample is denoted by ERSS $_{2}$ and is $\left(Y_{[1] 1}, Y_{[n] 2}, Y_{[1] 3} \ldots, Y_{[n] n-1}, \frac{\left(Y_{[1] n}+Y_{[n] n}\right)}{2}\right)$.

b) For the measure of the $n^{\text {th }}$ unit, choose $Y$ associated with median $X_{\left(\frac{n+1}{2}\right)}$ of $n^{\text {th }}$ sample. The selected sample will be denoted by $\mathrm{ERSS}_{3}$ and is given by $\left(Y_{[1] 1}, Y_{[n] 2}, Y_{[1] 3} \ldots, Y_{[n] n-1}, Y_{\left[\frac{n+1}{2}\right] n}\right)$

Thus, the samples under ERSS scheme in different situations can be represented as,

$$
\text { ERSS }_{i}= \begin{cases}Y_{[1] 1}, Y_{[n] 2}, Y_{[1] 3} \ldots, Y_{[1] n-1}, Y_{[n] n} & \text { if } i=1 \text { ( } \text { even) } \\ Y_{[1] 1}, Y_{[n] 2}, Y_{[1] 3} \ldots, Y_{[n] n-1}, \frac{\left(Y_{[1] n}+Y_{[n] n}\right)}{2} & \text { if } i=2(n \text { odd }) \\ Y_{[1] 1}, Y_{[n] 2}, Y_{[1] 3} \ldots, Y_{[n] n-1}, Y_{\left[\frac{n+1}{2}\right] n} & \text { if } i=3(n \text { odd })\end{cases}
$$

The estimator of $\theta_{2}$ using ERSS is defined as,

$$
\hat{\theta}_{2, \text { ERSS }_{i}}= \begin{cases}\frac{2}{n} \sum_{r=1}^{n / 2}\left(Y_{[1] 2 r-1}+Y_{[n] 2 r}\right) & \text { if } i=1 \\ \frac{2}{n}\left(Y_{[1] 1}+Y_{[n] 2}+Y_{[1] 3}+\cdots+Y_{[n] n-1}+\frac{Y_{[1] n}+Y_{[n] n}}{2}\right) & \text { if } i=2 \\ \frac{2}{n}\left(Y_{[1] 1}+Y_{[n] 2}+Y_{[1] 3}+\cdots+Y_{[n] n-1}+Y_{[(n+1) / 2] n}\right) & \text { if } i=3\end{cases}
$$

The variance of ERSS estimator of $\theta_{2}$ is, 


$$
\operatorname{Var}\left(\hat{\theta}_{2, E R S S_{i}}\right)= \begin{cases}\frac{4 \theta_{2}^{2} \delta_{1}}{n} & \text { if } i=1 \\ \frac{2}{n^{2}}\left((2 n-1) \theta_{2}^{2} \delta_{1}+\operatorname{cov}\left(Y_{[1] n}, Y_{[n] n}\right)\right) & \text { if } i=2 \\ \frac{\theta_{2}^{2}}{3 n^{2}}\left(12(n-1) \delta_{1}+1\right) & \text { if } i=3\end{cases}
$$

where $\delta_{1}=\frac{1}{12}-\left(\alpha m p \frac{B(m, m+n-1)}{B(1, n)} \frac{n-1}{2 m+n-1} \frac{B(p, p+1)}{2 p+1}\right)^{2}$.

Note that, in ERSS 1 and $\mathrm{ERSS}_{3}$, the selected elements are all independent. Hence their variance is free of any of the covariance terms while $\operatorname{Var}\left(\hat{\theta}_{2, E R S S_{2}}\right)$ involves $\operatorname{cov}\left(Y_{[1] n}, Y_{[n] n}\right)$ which is given by Scaria and Thomas (2008).

Now we summarize the efficiency of ERSS estimators of $\theta_{2}$ with other estimators in the following.

$$
\begin{aligned}
& e_{7}=R E\left(\hat{\theta}_{2, E R S S_{1}} \mid \hat{\theta}_{2, R S S}\right)=\frac{\sum_{r=1}^{n} \delta_{r}}{n \delta_{1}}, \\
& e_{8}=R E\left(\hat{\theta}_{2, E R S S_{1}} \mid \hat{\theta}_{2, B L U E}\right)=\frac{n}{4 \delta_{1} \sum_{r=1}^{n} \xi_{r}^{2} / \delta_{r}}, \\
& e_{9}=R E\left(\hat{\theta}_{2, E R S S_{1}} \mid \hat{\theta}_{2, L R S S}\right)=\frac{1}{4 \xi_{1}^{2}}, \\
& e_{10}=R E\left(\hat{\theta}_{2, E R S S_{1}} \mid \hat{\theta}_{2, U R S S}\right)=\frac{1}{4 \xi_{n}^{2}}, \\
& e_{11}=R E\left(\hat{\theta}_{2, E R S S_{3}} \mid \hat{\theta}_{2, R S S}\right)=\frac{12 \sum_{r=1}^{n} \delta_{r}}{12(n-1) \delta_{1}+1}, \\
& e_{12}=R E\left(\hat{\theta}_{2, E R S S_{3}} \mid \hat{\theta}_{2, B L U E}\right)=\frac{3 n^{2}}{\left(12(n-1) \delta_{1}+1\right)\left(\sum_{r=1}^{n} \xi_{r}^{2} / \delta_{r}\right)}, \\
& e_{13}=R E\left(\hat{\theta}_{2, E R S S_{3}} \mid \hat{\theta}_{2, L R S S}\right)=\frac{3 n \delta_{1}}{\xi_{1}^{2}\left(12(n-1) \delta_{1}+1\right)},
\end{aligned}
$$

and $\quad e_{14}=R E\left(\hat{\theta}_{2, E R S S_{3}} \mid \hat{\theta}_{2, U R S S}\right)=\frac{3 n \delta_{n}}{\xi_{n}^{2}\left(12(n-1) \delta_{n}+1\right)}$.

\subsection{MERSS estimation}

Al-Odat and Al-Saleh (2001) introduced the modified RSS scheme based on the concept of varied sample set size RSS and is known as MERSS. The steps to get MERSS sample from bivariate distribution given by Al-Saleh and Al-Ananbeh (2007) are as follows.

i) Select $k$ simple random sample sets of size $1,2, \ldots, k$ from bivariate distribution. Then identify by judgement the maximum of each set with respect to $X$-variate and measure the $Y$-variate associated with this $X$.

ii) Repeat step (i), but for minimum.

iii) Repeat the above two steps $l$ times, if necessary, until the desired sample size $n=2 l k$ is obtained.

With $l=1$, let $\left\{\left(X_{(r) r}, Y_{[r] r}\right),\left(X_{(1) r}^{\prime}, Y_{[1] r}^{\prime}\right) ; r=1,2, \ldots, k\right\}$ be the $n=2 k$ pairs that are obtained using above procedure where $X_{(r) r}=\max \left\{X_{r 1}, X_{r 2}, \ldots, X_{r r}\right\}$ for the samples 
selected in step (i) and $X_{(1) r}^{\prime}=\min \left\{X_{r 1}^{\prime}, X_{r 2}^{\prime}, \ldots, X_{r r}^{\prime}\right\}$ are the samples selected in step (ii), $r=1,2, \ldots ., k$. Note that the pairs of these samples are independent but not identically distributed as sample size varies. The set $\left(Y_{[1] 1}, Y_{[2] 2}, \ldots, Y_{[k] k}, Y_{[1] 1}^{\prime}, Y_{[1] 2}^{\prime}, \ldots, Y_{[1] k}^{\prime}\right)$ constitues the MERSS sample of size $n=2 k$ from $M T B G U$ distribution. An unbiased estimator of $\theta_{2}$ based on MERSS is given by,

$$
\hat{\theta}_{2, M E R S S}=\frac{1}{k} \sum_{r=1}^{k}\left(Y_{[r] r}+Y_{[1] r}^{\prime}\right)
$$

Now the variance of $Y_{[r] r}$ and $Y_{[1] r}^{\prime}$ are given by,

$$
\operatorname{Var}\left(Y_{[r] r}\right)=\theta_{2}^{2} \delta_{r}^{*}, \quad \operatorname{Var}\left(Y_{[1] r}^{\prime}\right)=\theta_{2}^{2} \delta_{1}^{*},
$$

where $\delta_{1}^{*}=\left.\delta_{1}\right|_{r=1, n=r}=\delta_{r}^{*}$.

The variance of $\hat{\theta}_{2, M E R S S}$ is,

$$
\operatorname{Var}\left(\hat{\theta}_{2, M E R S S}\right)=\frac{8 \theta_{2}^{2}}{n^{2}}\left(\sum_{r=1}^{n / 2} \delta_{1}^{*}\right)
$$

The efficiency of $\hat{\theta}_{2, M E R S S}$ over $\hat{\theta}_{2, R S S}$ and $\hat{\theta}_{2, S R S}$ are given as,

$$
R E\left(\hat{\theta}_{2, M E R S S} \mid \hat{\theta}_{2, R S S}\right)=\frac{\sum_{r=1}^{n} \delta_{r}}{2 \sum_{r=1}^{n / 2} \delta_{1}^{*}}, \quad R E\left(\hat{\theta}_{2, M E R S S} \mid \hat{\theta}_{2, S R S}\right)=\frac{n}{24 \sum_{r=1}^{n / 2} \delta_{1}^{*}}
$$

It is observed that $\hat{\theta}_{2, M E R S S}$ is more efficient than $\hat{\theta}_{2, S R S}$. The performance of $\hat{\theta}_{2, M E R S S}$ with respect to $\hat{\theta}_{2, R S S}$ depends on all the parameter values and $n$. To study the performance of the estimators with respect to parameters, we evaluate the efficiencies $e_{j}, j=1,2, \ldots, 14$ for different sample sizes in Section 5.

\section{Estimator of scale parameter based on censored RSS}

An example discussed by Bain (1978) about the oil pollution of sea water, uses the tar deposit in the nearly seashore as auxiliary variable. If there is no tar deposit at the seashore, then the corresponding sea sample will be treated as censored as these units cannot be measured. For ranking on $X$ observations in a sample, the censored units are assumed to have distinct and consecutive lower ranks and the remaining units are ranked with the next higher ranks in a natural order. If in this censored scheme of RSS, $k$ units are censored, then we may represent the RSS observations in RSS as $\rho_{1} Y_{[1] 1}, \rho_{2} Y_{[2] 2}, \ldots, \rho_{n} Y_{[n] n}$ where

$$
\rho_{i}= \begin{cases}0 & \text { if the } i^{t h} \text { unit is censored } \\ 1 & \text { otherwise }\end{cases}
$$

such that $\sum_{i=1}^{n} \rho_{i}=n-k$.

In this case the censored RSS mean $\bar{y}_{R S S}^{c}$ is defined as,

$$
\bar{y}_{R S S}^{c}=\frac{\sum_{i=1}^{n} \rho_{i} Y_{[i] i}}{n-k} .
$$


It may be noted that $\rho_{i}=0$ need not occur in a natural order for $i=1,2, \ldots, n$. Let the integers $n_{1}, n_{2}, \ldots, n_{n-k}$ be such that $1 \leq n_{1}<n_{2}<\cdots<n_{n-k} \leq n$ and $\rho_{n_{i}}=1$. Then,

$$
E\left[\bar{y}_{R S S}^{c}\right]=\frac{\theta_{2}}{n-k} \sum_{i=1}^{n-k} \xi_{n_{i}}
$$

where $\xi_{n_{i}}=\frac{1}{2}-\alpha m p \frac{B\left(m+n_{i}-1, m+n-n_{i}\right)}{B\left(n_{i}, n-n_{i}+1\right)} \frac{n-2 n_{i}+1}{2 m+n-1} \frac{B(p, p+1)}{2 p+1}$

Even though the RSS mean in censored case is not an unbiased estimator for $\theta_{2}$, we can construct an unbiased estimator based on $\bar{y}_{R S S}^{c}$. Let $Y_{\left[n_{i}\right] n_{i}}, i=1,2, \ldots, n-k$ be the censored RSS observations. When $m, p$ and $\alpha$ are known, an unbiased estimator $\hat{\theta}_{2, R S S}^{c}(k)$ of $\theta_{2}$ based on censored RSS of size $k$ and its variance is given by,

$$
\hat{\theta}_{2, R S S}^{c}(k)=\frac{(n-k) \bar{y}_{R S S}^{c}}{\sum_{i=1}^{n-k} \xi_{n_{i}}} \quad \text { and } \quad \operatorname{Var}\left(\hat{\theta}_{2, R S S}^{c}(k)\right)=\theta_{2}^{2} \frac{\sum_{i=1}^{n-k} \delta_{n_{i}}}{\left(\sum_{i=1}^{n-k} \xi_{n_{i}}\right)^{2}} \text {, }
$$

where $\delta_{n_{i}}=\frac{1}{12}-\left(\alpha m p \frac{B\left(m+n_{i}-1, m+n-n_{i}\right)}{B\left(n_{i}, n-n_{i}+1\right)} \frac{n-2 n_{i}+1}{2 m+n-1} \frac{B(p, p+1)}{2 p+1}\right)^{2}, i=1,2, \ldots, n-k$.

We now propose the BLUE of $\theta_{2}$ based on the censored RSS. Let $\boldsymbol{Y}_{[n]}(k)=$ $\left(Y_{\left[n_{1}\right] n_{1}} \quad Y_{\left[n_{2}\right] n_{2}} \quad \cdots \quad Y_{\left[n_{n-k}\right] n_{n-k}}\right)$ ' be the vector of observations in censored RSS. The mean vector and the dispersion matrix of $\boldsymbol{Y}_{[\boldsymbol{n}]}(k)$ are given by,

$$
\begin{aligned}
& E\left(\boldsymbol{Y}_{[\boldsymbol{n}]}(k)\right)=\theta_{2} \xi(\boldsymbol{k}), \\
& D\left(\boldsymbol{Y}_{[\boldsymbol{n}]}(k)\right)=\theta_{2}^{2} G(k),
\end{aligned}
$$

where $\boldsymbol{\xi}(\boldsymbol{k})=\left(\begin{array}{llll}\xi_{n_{1}} & \xi_{n_{2}} & \cdots & \xi_{n_{n-k}}\end{array}\right)^{\prime}$ and $G(k)=\operatorname{diag}\left(\delta_{n_{1}}, \delta_{n_{2}}, \ldots, \delta_{n_{n-k}}\right)$.

If $m, p$ and $\alpha$ involved in $\boldsymbol{\xi}(\boldsymbol{k})$ and $G(k)$ are known, then (3) and (4) defines a generalized Gauss-Markov set up and hence the BLUE $\tilde{\theta}_{2, R S S}^{c}(k)$ of $\theta_{2}$ is obtained as,

$$
\tilde{\theta}_{2, R S S}^{c}(k)=\left(\xi(\boldsymbol{k})^{\prime} G(k)^{-1} \boldsymbol{\xi}(\boldsymbol{k})\right)^{-1} \xi(\boldsymbol{k})^{\prime} G(k)^{-1} \boldsymbol{Y}_{[\boldsymbol{n}]}(k),
$$

and $\operatorname{Var}\left(\tilde{\theta}_{2, R S S}^{c}(k)\right)=\left(\xi(\boldsymbol{k})^{\prime} G(k)^{-1} \xi(\boldsymbol{k})\right)^{-1} \theta_{2}^{2}$

Simplifying the expression from (5) and (6), we get,

$$
\begin{aligned}
& \tilde{\theta}_{2, R S S}^{c}(k)=\sum_{i=1}^{n-k} a_{n_{i}} Y_{\left[n_{i}\right] n_{i},} \\
& \operatorname{Var}\left(\tilde{\theta}_{2, R S S}^{c}(k)\right)=\frac{\theta_{2}^{2}}{\sum_{i=1}^{n-k}\left(\xi_{n_{i}}^{2} / \delta_{n_{i}}\right)} .
\end{aligned}
$$

where $\quad a_{m_{i}}=\frac{\xi_{n_{i i}} / \delta_{n_{i_{i}}}}{\sum_{i=1}^{n-k} \xi_{n_{i}}^{2} / \delta_{n_{i}}}$. 
Remark 1: The efficiencies developed in Section 3 are in terms of association parameter $\alpha$ and $m, p$. An estimator of $\alpha$ can be obtained using sample correlation coefficient. Scaria and Thomas (2008) obtained correlation coefficient for $(X, Y) \sim \operatorname{MTBGU}\left(\theta_{1}, \theta_{2}, m, p, \alpha\right)$ distribution and is given by,

$$
\rho=12 \alpha f(m, p)
$$

where $f(m, p)=B(m+1, m+1) B(p+1, p+1)$.

We propose an estimator of $\alpha$ based on sample correlation coefficient $\hat{\rho}$ of the observations $\left(X_{(r) r}, Y_{[r] r}\right), r=1,2, \ldots, n$ assuming $m$ and $p$ known. This estimator is in similar lines to the estimator of $\alpha$ proposed by Chacko and Thomos (2008) for Morgenstern type bivariate exponential distribution and is as follows.

$$
\hat{\alpha}=\frac{\hat{\rho}}{12 f(m, p)} \quad \text { if }-12 f(m, p) \leq \hat{\rho} \leq 12 f(m, p)
$$

In the following we summarize the range of association parameter $\alpha$ and its estimator

\begin{tabular}{|c|c|c|c|}
\hline Distribution & Range of $\alpha$ & $\rho$ & Estimator of $\alpha$ \\
\hline $\begin{array}{c}M T B G U\left(\theta_{1}, \theta_{2}, 1,1, \alpha\right) \\
\text { (i.e. Morgenstern type } \\
\text { bivariate uniform) }\end{array}$ & $\begin{array}{l}{[-1,1]} \\
\text { (Morgenstern } \\
(1956))\end{array}$ & $\frac{\alpha}{3}$ & $\begin{array}{l}\hat{\alpha}=\left\{\begin{array}{lc}-1 & \text { if } \hat{\rho}<-\frac{1}{3} \\
3 \hat{\rho} \text { if }-\frac{1}{3} \leq \hat{\rho} \leq \frac{1}{3} & \\
1 & \text { if } \hat{\rho}>\frac{1}{3}\end{array}\right. \\
\text { (proposed by Tahmasebi and Jafari } \\
\text { (2012)) }\end{array}$ \\
\hline $\operatorname{MTBGU}(1,1, m, p, \alpha)$ & $\begin{array}{c}{\left[-\alpha_{u}, \alpha_{u}\right]} \\
(\text { Tomas and } \\
\text { Veena }(2011)) \\
\end{array}$ & \multirow[t]{2}{*}{$12 f(m, p)$} & \multirow[t]{2}{*}{$\hat{\alpha}=\frac{\hat{\rho}}{12 f(m, p)} \quad$ if $|\hat{\rho}| \leq 12 f(m, p)$} \\
\hline $\operatorname{MTBGU}\left(\theta_{1}, \theta_{2}, m, p, \alpha\right)$ & {$\left[-\alpha_{u}^{*}, \alpha_{u}^{*}\right]$} & & \\
\hline
\end{tabular}
for the MTBGU distribution in different situations.

Here $\alpha_{u}=\frac{1}{m p\left[\left(\frac{\sqrt{2}}{\sqrt{4 m-2}}\right)\left(\frac{1}{4}-\frac{1}{2(4 m-2)}\right)^{m-1}\right]\left[\left(\frac{\sqrt{2}}{\sqrt{4 p-2}}\right)\left(\frac{1}{4}-\frac{1}{2(4 p-2)}\right)^{p-1}\right]}$ and $\alpha_{u}^{*}$ is function of $m$ and $p$ so that $F(x, y)$ forms the distribution function of MTBGU distribution.

\section{Numerical study}

In this section, we evaluate the efficiencies $e_{j}, j=1,2, \ldots, 14$ based on different RSS schemes as RSS, LRSS, URSS and ERSS. The efficiencies are evaluated for $n=$ $5,10,15,20, \alpha= \pm 0.25, \pm 0.50, \pm 0.75$ and for different values of $m$ and $p$. Table 1 presents the efficiencies for $m=1, p=2$, Table 2 considers $m=2, p=1$ and Table 3 shows these efficiencies for $m=1, p=1.5$. The efficiencies $e_{7}, e_{8}, e_{9}, e_{10}\left(e_{11}, e_{12}, e_{13}, e_{14}\right)$ represent efficiency of $\hat{\theta}_{2, E R S S_{1}}\left(\hat{\theta}_{2, E R S S_{3}}\right)$ over $\hat{\theta}_{2, R S S}, \hat{\theta}_{2, B L U E}, \hat{\theta}_{2, L R S S}$ and $\hat{\theta}_{2, U R S S}$ respectively, when sample size is even (odd). 
Table 1: The efficiencies $e_{j}, j=1,2, \ldots, 14$ for $m=1$ and $p=2$

\begin{tabular}{|c|c|c|c|c|c|c|c|c|c|c|c|c|c|c|c|}
\hline $\boldsymbol{n}$ & $\alpha$ & $e_{1}$ & $e_{2}$ & $e_{3}$ & $e_{4}$ & $e_{5}$ & $e_{6}$ & $e_{7}$ & $e_{8}$ & $e_{9}$ & $e_{10}$ & $e_{11}$ & $e_{12}$ & $e_{13}$ & $e_{14}$ \\
\hline \multirow{6}{*}{5} & -0.75 & 1.0017 & 1.0006 & 1.0696 & 1.0690 & 0.9360 & 0.9355 & -- & -- & -- & -- & 1.0010 & 1.0004 & 0.9359 & 1.0694 \\
\hline & -0.50 & 1.0007 & 1.0002 & 1.0457 & 1.0455 & 0.9568 & 0.9565 & -- & -- & -- & -- & 1.0004 & 1.0002 & 0.9567 & 1.0457 \\
\hline & -0.25 & 1.0002 & 1.0001 & 1.0225 & 1.0225 & 0.9781 & 0.9780 & -- & -- & -- & -- & 1.0001 & 1.0000 & 0.9781 & 1.0225 \\
\hline & 0.25 & 1.0002 & 1.0001 & 0.9781 & 0.9780 & 1.0225 & 1.0225 & -- & -- & -- & -- & 1.0001 & 1.0000 & 1.0225 & 0.9781 \\
\hline & 0.50 & 1.0007 & 1.0002 & 0.9568 & 0.9565 & 1.0457 & 1.0455 & -- & -- & -- & -- & 1.0004 & 1.0002 & 1.0457 & 0.9567 \\
\hline & 0.75 & 1.0017 & 1.0006 & 0.9360 & 0.9355 & 1.0696 & 1.0690 & -- & -- & -- & -- & 1.0010 & 1.0004 & 1.0694 & 0.9359 \\
\hline \multirow{6}{*}{10} & -0.75 & 1.0020 & 1.0007 & 1.0867 & 1.0860 & 0.9226 & 0.9220 & 1.0030 & 1.0023 & 0.9229 & 1.0871 & -- & -- & -- & -- \\
\hline & -0.50 & 1.0009 & 1.0003 & 1.0567 & 1.0564 & 0.9475 & 0.9472 & 1.0013 & 1.0010 & 0.9476 & 1.0569 & -- & -- & -- & -- \\
\hline & -0.25 & 1.0002 & 1.0001 & 1.0278 & 1.0277 & 0.9732 & 0.9732 & 1.0003 & 1.0003 & 0.9733 & 1.0278 & -- & -- & -- & -- \\
\hline & 0.25 & 1.0002 & 1.0001 & 0.9732 & 0.9732 & 1.0278 & 1.0277 & 1.0003 & 1.0003 & 1.0278 & 0.9733 & -- & -- & -- & -- \\
\hline & 0.50 & 1.0009 & 1.0003 & 0.9475 & 0.9472 & 1.0567 & 1.0564 & 1.0013 & 1.0010 & 1.0569 & 0.9476 & -- & -- & -- & -- \\
\hline & 0.75 & 1.0020 & 1.0007 & 0.9226 & 0.9220 & 1.0867 & 1.0860 & 1.0030 & 1.0023 & 1.0871 & 0.9229 & -- & -- & -- & -- \\
\hline \multirow{6}{*}{15} & -0.75 & 1.0022 & 1.0007 & 1.0933 & 1.0925 & 0.9177 & 0.9170 & -- & -- & -- & -- & 1.0032 & 1.0025 & 0.9176 & 1.0932 \\
\hline & -0.50 & 1.0010 & 1.0003 & 1.0609 & 1.0605 & 0.9440 & 0.9437 & -- & -- & -- & -- & 1.0014 & 1.0011 & 0.9440 & 1.0608 \\
\hline & -0.25 & 1.0002 & 1.0001 & 1.0298 & 1.0297 & 0.9714 & 0.9714 & -- & -- & -- & -- & 1.0004 & 1.0003 & 0.9714 & 1.0298 \\
\hline & 0.25 & 1.0002 & 1.0001 & 0.9714 & 0.9714 & 1.0298 & 1.0297 & -- & -- & -- & -- & 1.0004 & 1.0003 & 1.0298 & 0.9714 \\
\hline & 0.50 & 1.0010 & 1.0003 & 0.9440 & 0.9437 & 1.0609 & 1.0605 & -- & -- & -- & -- & 1.0014 & 1.0011 & 1.0608 & 0.9440 \\
\hline & 0.75 & 1.0022 & 1.0007 & 0.9177 & 0.9170 & 1.0933 & 1.0925 & -- & -- & -- & -- & 1.0032 & 1.0025 & 1.0932 & 0.9176 \\
\hline \multirow{6}{*}{20} & -0.75 & 1.0023 & 1.0008 & 1.0968 & 1.0960 & 0.9151 & 0.9144 & 1.0039 & 1.0031 & 0.9153 & 1.0970 & -- & -- & -- & -- \\
\hline & -0.50 & 1.0010 & 1.0003 & 1.0631 & 1.0627 & 0.9422 & 0.9419 & 1.0017 & 1.0014 & 0.9423 & 1.0632 & -- & -- & -- & -- \\
\hline & -0.25 & 1.0003 & 1.0001 & 1.0308 & 1.0307 & 0.9705 & 0.9704 & 1.0004 & 1.0003 & 0.9705 & 1.0309 & -- & -- & -- & -- \\
\hline & 0.25 & 1.0003 & 1.0001 & 0.9705 & 0.9704 & 1.0308 & 1.0307 & 1.0004 & 1.0003 & 1.0309 & 0.9705 & -- & -- & -- & -- \\
\hline & 0.50 & 1.0010 & 1.0003 & 0.9422 & 0.9419 & 1.0631 & 1.0627 & 1.0017 & 1.0014 & 1.0632 & 0.9423 & -- & -- & -- & -- \\
\hline & 0.75 & 1.0023 & 1.0008 & 0.9151 & 0.9144 & 1.0968 & 1.0960 & 1.0039 & 1.0031 & 1.097 & 0.9153 & -- & -- & -- & -- \\
\hline
\end{tabular}


Table 2: The efficiencies $e_{j}, j=1,2, \ldots, 14$ for $m=2$ and $p=1$

\begin{tabular}{|c|c|c|c|c|c|c|c|c|c|c|c|c|c|c|c|}
\hline $\boldsymbol{n}$ & $\alpha$ & $e_{1}$ & $e_{2}$ & $\boldsymbol{e}_{3}$ & $e_{4}$ & $e_{5}$ & $e_{6}$ & $e_{7}$ & $e_{8}$ & $e_{9}$ & $e_{10}$ & $e_{11}$ & $e_{12}$ & $e_{13}$ & $\boldsymbol{e}_{14}$ \\
\hline \multirow{6}{*}{5} & -0.75 & 1.0017 & 1.0006 & 1.0614 & 1.0608 & 0.9422 & 0.9417 & -- & -- & -- & -- & 1.0004 & 0.9998 & 0.9425 & 1.0617 \\
\hline & -0.50 & 1.0008 & 1.0003 & 1.0405 & 1.0402 & 0.9611 & 0.9609 & -- & -- & -- & -- & 1.0002 & 0.9999 & 0.9612 & 1.0406 \\
\hline & -0.25 & 1.0002 & 1.0001 & 1.0200 & 1.0200 & 0.9804 & 0.9803 & -- & -- & -- & -- & 1.0000 & 1.0000 & 0.9804 & 1.0201 \\
\hline & 0.25 & 1.0002 & 1.0001 & 0.9804 & 0.9803 & 1.0200 & 1.0200 & -- & -- & -- & -- & 1.0000 & 1.0000 & 1.0201 & 0.9804 \\
\hline & 0.50 & 1.0008 & 1.0003 & 0.9611 & 0.9609 & 1.0405 & 1.0402 & -- & -- & -- & -- & 1.0002 & 0.9999 & 1.0406 & 0.9612 \\
\hline & 0.75 & 1.0017 & 1.0006 & 0.9422 & 0.9417 & 1.0614 & 1.0608 & -- & -- & -- & -- & 1.0004 & 0.9998 & 1.0617 & 0.9425 \\
\hline \multirow{6}{*}{10} & -0.75 & 1.0024 & 1.0008 & 1.0528 & 1.0520 & 0.9480 & 0.9472 & 0.9997 & 0.9989 & 0.9495 & 1.0546 & -- & -- & -- & -- \\
\hline & -0.50 & 1.0011 & 1.0004 & 1.0351 & 1.0348 & 0.9652 & 0.9649 & 0.9999 & 0.9995 & 0.9659 & 1.0359 & -- & -- & -- & -- \\
\hline & -0.25 & 1.0003 & 1.0001 & 1.0175 & 1.0174 & 0.9826 & 0.9825 & 1.0000 & 0.9999 & 0.9827 & 1.0177 & -- & -- & -- & -- \\
\hline & 0.25 & 1.0003 & 1.0001 & 0.9826 & 0.9825 & 1.0175 & 1.0174 & 1.0000 & 0.9999 & 1.0177 & 0.9827 & -- & -- & -- & -- \\
\hline & 0.50 & 1.0011 & 1.0004 & 0.9652 & 0.9649 & 1.0351 & 1.0348 & 0.9999 & 0.9995 & 1.0359 & 0.9659 & -- & -- & -- & -- \\
\hline & 0.75 & 1.0024 & 1.0008 & 0.9480 & 0.9472 & 1.0528 & 1.0520 & 0.9997 & 0.9989 & 1.0546 & 0.9495 & -- & -- & -- & -- \\
\hline \multirow{6}{*}{15} & -0.75 & 1.0027 & 1.0009 & 1.0420 & 1.0411 & 0.9563 & 0.9555 & -- & -- & -- & -- & 0.9986 & 0.9977 & 0.9584 & 1.0442 \\
\hline & -0.50 & 1.0012 & 1.0004 & 1.0282 & 1.0278 & 0.9711 & 0.9707 & -- & -- & -- & -- & 0.9994 & 0.9990 & 0.972 & 1.0292 \\
\hline & -0.25 & 1.0003 & 1.0001 & 1.0142 & 1.0141 & 0.9856 & 0.9855 & -- & -- & -- & -- & 0.9998 & 0.9997 & 0.9858 & 1.0144 \\
\hline & 0.25 & 1.0003 & 1.0001 & 0.9856 & 0.9855 & 1.0142 & 1.0141 & -- & -- & -- & -- & 0.9998 & 0.9997 & 1.0144 & 0.9858 \\
\hline & 0.50 & 1.0012 & 1.0004 & 0.9711 & 0.9707 & 1.0282 & 1.0278 & -- & -- & -- & -- & 0.9994 & 0.9990 & 1.0292 & 0.9720 \\
\hline & 0.75 & 1.0027 & 1.0009 & 0.9563 & 0.9555 & 1.0420 & 1.0411 & -- & -- & -- & -- & 0.9986 & 0.9977 & 1.0442 & 0.9584 \\
\hline \multirow{6}{*}{20} & -0.75 & 1.0029 & 1.0010 & 1.0341 & 1.0331 & 0.9627 & 0.9618 & 0.9981 & 0.9972 & 0.9652 & 1.0367 & -- & -- & -- & -- \\
\hline & -0.50 & 1.0013 & 1.0004 & 1.0231 & 1.0227 & 0.9755 & 0.9751 & 0.9992 & 0.9987 & 0.9766 & 1.0243 & -- & -- & -- & -- \\
\hline & -0.25 & 1.0003 & 1.0001 & 1.0117 & 1.0116 & 0.9879 & 0.9878 & 0.9998 & 0.9997 & 0.9882 & 1.0120 & -- & -- & -- & -- \\
\hline & 0.25 & 1.0003 & 1.0001 & 0.9879 & 0.9878 & 1.0117 & 1.0116 & 0.9998 & 0.9997 & 1.0120 & 0.9882 & -- & -- & -- & -- \\
\hline & 0.50 & 1.0013 & 1.0004 & 0.9755 & 0.9751 & 1.0231 & 1.0227 & 0.9992 & 0.9987 & 1.0243 & 0.9766 & -- & -- & -- & -- \\
\hline & 0.75 & 1.0029 & 1.0010 & 0.9627 & 0.9618 & 1.0341 & 1.0331 & 0.9981 & 0.9972 & 1.0367 & 0.9652 & -- & -- & -- & -- \\
\hline
\end{tabular}


Table 3: The efficiencies $e_{j}, j=1,2, \ldots, 14$ for $m=1$ and $p=1.5$

\begin{tabular}{|c|c|c|c|c|c|c|c|c|c|c|c|c|c|c|c|}
\hline$n$ & $\alpha$ & $e_{1}$ & $e_{2}$ & $e_{3}$ & $e_{4}$ & $e_{5}$ & $e_{6}$ & $e_{7}$ & $e_{8}$ & $e_{9}$ & $e_{10}$ & $e_{11}$ & $\boldsymbol{e}_{12}$ & $e_{13}$ & $e_{14}$ \\
\hline \multirow{6}{*}{5} & -0.75 & 1.0082 & 1.0028 & 1.1622 & 1.1590 & 0.8653 & 0.8629 & -- & -- & -- & -- & 1.0049 & 1.0022 & 0.8647 & 1.1614 \\
\hline & -0.50 & 1.0036 & 1.0012 & 1.1046 & 1.1032 & 0.9075 & 0.9064 & -- & -- & -- & -- & 1.0022 & 1.0010 & 0.9073 & 1.1043 \\
\hline & -0.25 & 1.0009 & 1.0003 & 1.0506 & 1.0503 & 0.9524 & 0.9521 & -- & -- & -- & -- & 1.0005 & 1.0002 & 0.9523 & 1.0506 \\
\hline & 0.25 & 1.0009 & 1.0003 & 0.9524 & 0.9521 & 1.0506 & 1.0503 & -- & -- & -- & -- & 1.0005 & 1.0002 & 1.0506 & 0.9523 \\
\hline & 0.50 & 1.0036 & 1.0012 & 0.9075 & 0.9064 & 1.1046 & 1.1032 & -- & -- & -- & -- & 1.0022 & 1.0010 & 1.1043 & 0.9073 \\
\hline & 0.75 & 1.0082 & 1.0028 & 0.8653 & 0.8629 & 1.1622 & 1.1590 & -- & -- & -- & -- & 1.0049 & 1.0022 & 1.1614 & 0.8647 \\
\hline \multirow{6}{*}{10} & -0.75 & 1.0101 & 1.0034 & 1.2066 & 1.2025 & 0.8397 & 0.8369 & 1.0149 & 1.0114 & 0.8411 & 1.2086 & -- & -- & -- & -- \\
\hline & -0.50 & 1.0045 & 1.0015 & 1.1314 & 1.1298 & 0.8889 & 0.8876 & 1.0065 & 1.0050 & 0.8896 & 1.1323 & -- & -- & -- & -- \\
\hline & -0.25 & 1.0011 & 1.0004 & 1.0629 & 1.0625 & 0.9422 & 0.9418 & 1.0016 & 1.0012 & 0.9424 & 1.0631 & -- & -- & -- & -- \\
\hline & 0.25 & 1.0011 & 1.0004 & 0.9422 & 0.9418 & 1.0629 & 1.0625 & 1.0016 & 1.0012 & 1.0631 & 0.9424 & -- & -- & -- & -- \\
\hline & 0.50 & 1.0045 & 1.0015 & 0.8889 & 0.8876 & 1.1314 & 1.1298 & 1.0065 & 1.0050 & 1.1323 & 0.8896 & -- & -- & -- & -- \\
\hline & 0.75 & 1.0101 & 1.0034 & 0.8397 & 0.8369 & 1.2066 & 1.2025 & 1.0149 & 1.0114 & 1.2086 & 0.8411 & -- & -- & -- & -- \\
\hline \multirow{6}{*}{15} & -0.75 & 1.0108 & 1.0037 & 1.2241 & 1.2196 & 0.8306 & 0.8276 & -- & -- & -- & -- & 1.0159 & 1.0122 & 0.8299 & 1.2231 \\
\hline & -0.50 & 1.0048 & 1.0016 & 1.1418 & 1.1400 & 0.8821 & 0.8807 & -- & -- & -- & -- & 1.0070 & 1.0053 & 0.8819 & 1.1415 \\
\hline & -0.25 & 1.0012 & 1.0004 & 1.0675 & 1.0671 & 0.9384 & 0.9380 & -- & -- & -- & -- & 1.0017 & 1.0013 & 0.9384 & 1.0675 \\
\hline & 0.25 & 1.0012 & 1.0004 & 0.9384 & 0.9380 & 1.0675 & 1.0671 & -- & -- & -- & -- & 1.0017 & 1.0013 & 1.0675 & 0.9384 \\
\hline & 0.50 & 1.0048 & 1.0016 & 0.8821 & 0.8807 & 1.1418 & 1.1400 & -- & -- & -- & -- & 1.0070 & 1.0053 & 1.1415 & 0.8819 \\
\hline & 0.75 & 1.0108 & 1.0037 & 0.8306 & 0.8276 & 1.2241 & 1.2196 & -- & -- & -- & -- & 1.0159 & 1.0122 & 1.2231 & 0.8299 \\
\hline \multirow{6}{*}{20} & -0.75 & 1.0112 & 1.0038 & 1.2334 & 1.2288 & 0.8259 & 0.8228 & 1.0195 & 1.0156 & 0.8266 & 1.2344 & -- & -- & -- & -- \\
\hline & -0.50 & 1.0049 & 1.0017 & 1.1474 & 1.1455 & 0.8786 & 0.8772 & 1.0085 & 1.0069 & 0.879 & 1.1478 & -- & -- & -- & -- \\
\hline & -0.25 & 1.0012 & 1.0004 & 1.0700 & 1.0695 & 0.9365 & 0.9361 & 1.0021 & 1.0017 & 0.9366 & 1.0701 & -- & -- & -- & -- \\
\hline & 0.25 & 1.0012 & 1.0004 & 0.9365 & 0.9361 & 1.0700 & 1.0695 & 1.0021 & 1.0017 & 1.0701 & 0.9366 & -- & -- & -- & -- \\
\hline & 0.50 & 1.0049 & 1.0017 & 0.8786 & 0.8772 & 1.1474 & 1.1455 & 1.0085 & 1.0069 & 1.1478 & 0.8790 & -- & -- & -- & -- \\
\hline & 0.75 & 1.0112 & 1.0038 & 0.8259 & 0.8228 & 1.2334 & 1.2288 & 1.0195 & 1.0156 & 1.2344 & 0.8266 & -- & -- & -- & -- \\
\hline
\end{tabular}


Different trends can be seen in these efficiencies with respect to $n, \alpha, m$ and $p$. Some observations for the numerical work in Table 1, Table 2 and Table 3 are as follows.

i) $\hat{\theta}_{2, R S S}$ is more efficient than $\hat{\theta}_{2, S R S}$ and the efficiency $e_{1}$ increases with increase in $n$ and $|\alpha|$. Observe that for larger values of $m$ and $p, \hat{\theta}_{2, B L U E}$ and $\hat{\theta}_{2, R S S}$ are almost equally efficient.

ii) $\hat{\theta}_{2, L R S S}\left(\hat{\theta}_{2, B L U E}\right)$ is more efficient than $\hat{\theta}_{2, B L U E}\left(\hat{\theta}_{2, L R S S}\right)$ for $\alpha<0(\alpha>0)$ and efficiency $e_{4}$ increases with increase in $n$ for fixed $\alpha<0$. Same behavior is observed for the efficiency $e_{3}$ of $\hat{\theta}_{2, L R S S}$ over $\hat{\theta}_{2, R S S}$.

iii) $\hat{\theta}_{2, U R S S}\left(\hat{\theta}_{2, B L U E}\right)$ is more efficient than $\hat{\theta}_{2, B L U E}\left(\hat{\theta}_{2, U R S S}\right)$ for $\alpha>0(\alpha<0)$ and efficiency $e_{6}$ increases with increase in $n$ for fixed $\alpha>0$. Same behavior is observed for the efficiency $e_{5}$ of $\hat{\theta}_{2, U R S S}$ over $\hat{\theta}_{2, R S S}$.

iv) For $m=1$ and different $p$, we observe that $\hat{\theta}_{2, E R S S_{1}}$ and $\hat{\theta}_{2, E R S S_{3}}$ are efficient than $\hat{\theta}_{2, B L U E}$. For different combinations of $(m, p)$ we observe that $\hat{\theta}_{2, U R S S}\left(\hat{\theta}_{2, L R S S}\right)$ is more efficient than $\hat{\theta}_{2, E R S S_{1}}, \hat{\theta}_{2, E R S S_{3}}$ for $\alpha>0(\alpha<0)$ and $\hat{\theta}_{2, E R S S_{1}}, \hat{\theta}_{2, E R S S_{3}}$ are more efficient than $\hat{\theta}_{2, U R S S}\left(\hat{\theta}_{2, L R S S}\right)$ for $\alpha<0(\alpha>0)$.

v) Overall it is observed that for $\alpha<0, \hat{\theta}_{2, L R S S}$ and for $\alpha>0, \hat{\theta}_{2, U R S S}$ is most efficient than all other obtained estimators.

Now we present behavior of efficiencies of estimators under various schemes with respect to various parameters. Figure 1 presents the trends in efficiencies $\hat{\theta}_{2, L R S S}, \hat{\theta}_{2, U R S S}$ and $\hat{\theta}_{2, E R S S_{3}}$ over $\hat{\theta}_{2, R S S}$ across the various values of association parameter $\alpha$ when $m=1, p=$ 1.5 and $n=15$. Figure 2 presents these trends across $\alpha$ for $m=1, p=1.5$ and $n=10$.

Figure 1: Efficiencies $E_{B L U E}, E_{L R S S}, E_{U R S S}$ and $E_{E R S S_{3}}$ across $\alpha$ when $m=1, p=1.5$ and $n=15$

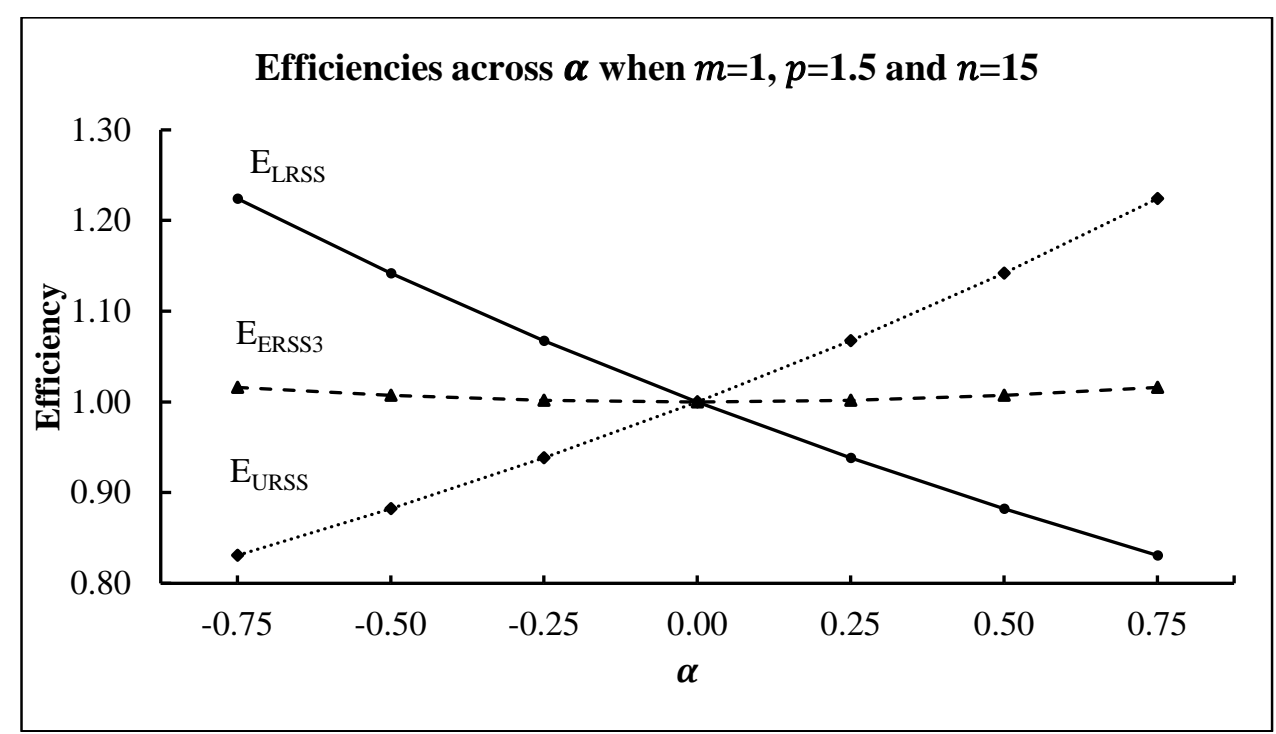


Figure 2: Efficiencies $E_{B L U E}, E_{L R S S}, E_{U R S S}$ and $E_{E R S S_{1}}$ across $\alpha$ when $m=1, p=1.5$ and $n=10$

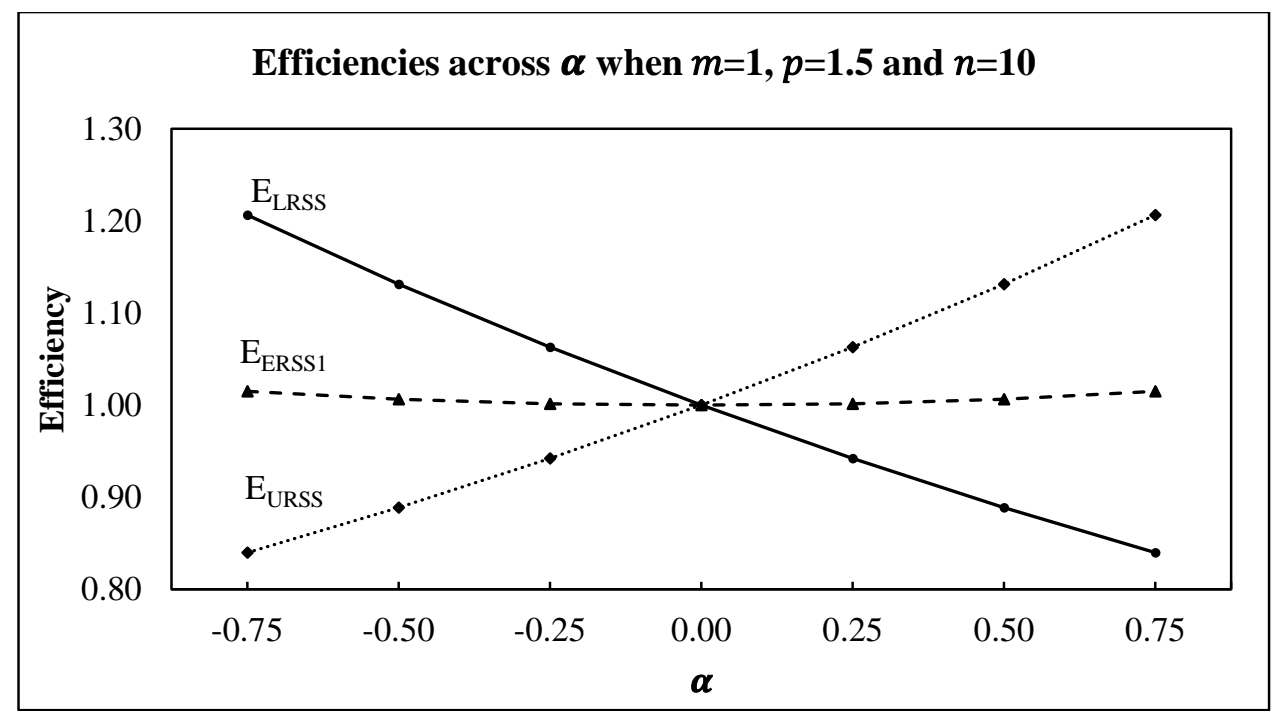

From Figure 1 and Figure 2, we observe that for $\alpha<0$, the performance of $\hat{\theta}_{2, L R S S}$ is the best and $E_{L R S S}$ decreases as $\alpha$ increases. While $\hat{\theta}_{2, U R S S}$ is most efficient among all other estimators when $\alpha>0$. Specifically the behaviour of $E_{U R S S}$ is exactly opposite to that of $E_{L R S S}$ with respect to $\alpha_{3}$. We observe that efficiencies $E_{B L U E}$ and $E_{E R S S_{i}}, i=1,3$ vary in a very small interval.

The variation in efficiencies of $\hat{\theta}_{2, L R S S}$ over $\hat{\theta}_{2, R S S}$ with respect to $n$ and $\alpha$ when $m=$ $1, p=1$ is presented in Figure 3 .

Figure 3: Efficiency $E_{L R S S}$ of $\widehat{\theta}_{2, L R S S}$ over $\widehat{\theta}_{2, R S S}$ with respect to $n$ and $\alpha$ when $m=1$ and $p=1$

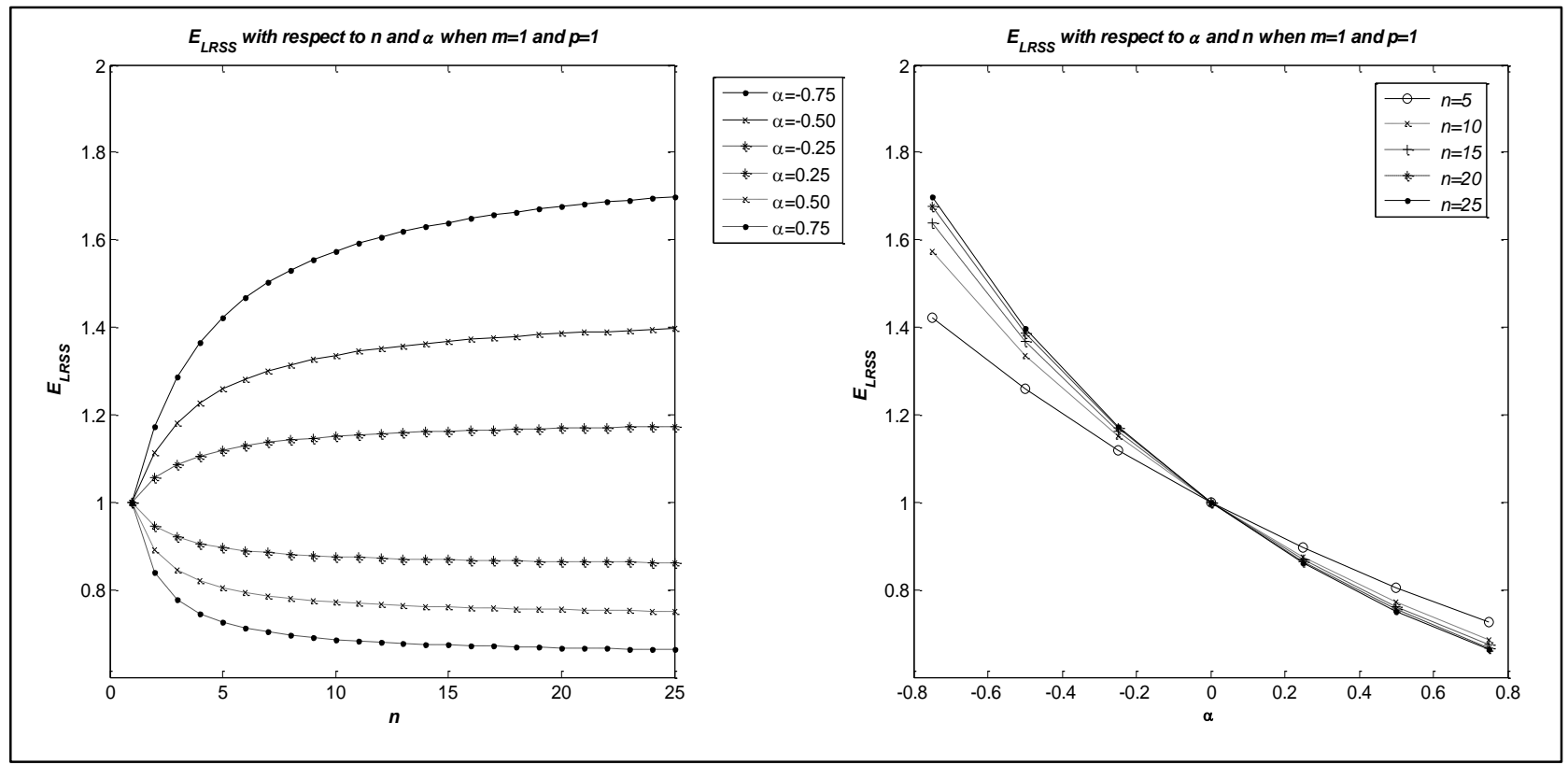

From Figure 3 we observe that for $\alpha<0$, efficiency $E_{\text {LRSS }}$ increases as $n$ increases and efficiency stabilize for larger $n$. We also observe that $E_{L R S S}$ decreases non-linearly as $\alpha$ increases from -0.8 to 0.8 . Now we study the behavior of $E_{U R S S}$ across $n$ and $\alpha$ when $m=2$ and $p=3$. The results are presented in Figure 4. 
Figure 4: Efficiency $E_{U R S S}$ of $\widehat{\theta}_{2, L R S S}$ over $\widehat{\theta}_{2, R S S}$ with respect to $n$ and $\alpha$ when $m=2$ and $p=3$

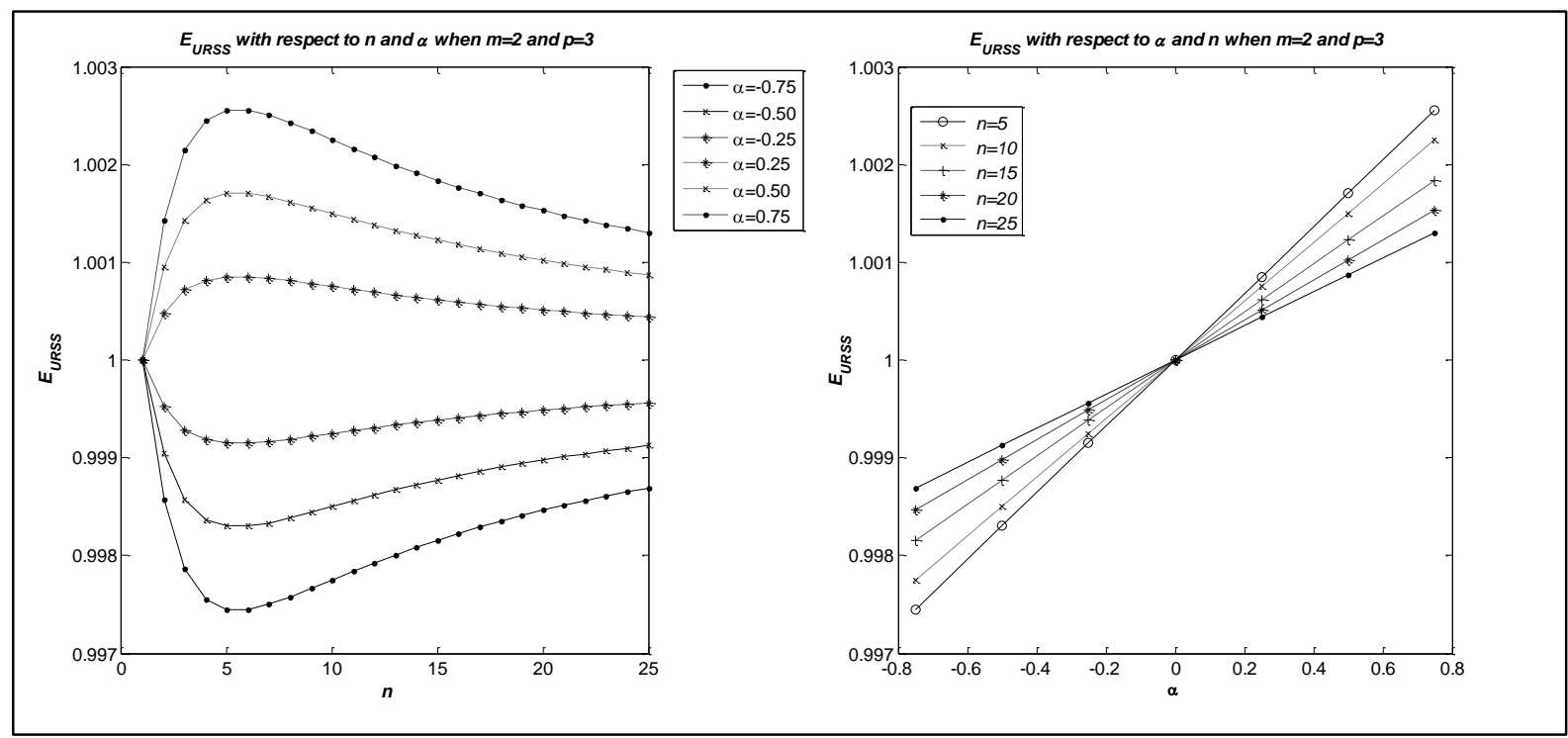

From Figure 4 we observe that for $\alpha>0$ efficiency $E_{U R S S}$ increases up to $n=5$ then it decreases and gets stabilize for larger $n$. We observe that $E_{U R S S}$ increases linearly as $\alpha$ increases from -0.8 to 0.8 .

Figure 5 presents the surface plot of efficiency $E_{L R S S}\left(E_{U R S S}\right)$ with respect to $m$ and $p$ for $\alpha=-0.5(0.25), n=10$.

Figure 5: Efficiency of $\widehat{\theta}_{2, L R S S}\left(E_{L R S S}\right)$ and $\widehat{\theta}_{2, U R S S}\left(E_{U R S S}\right)$ over $\widehat{\theta}_{2, R S S}$ for $\alpha=-0.5(\alpha=0.25)$

E_LRSS with respec to $m$ and $p$ when $a=-0.5$ and $n=10$

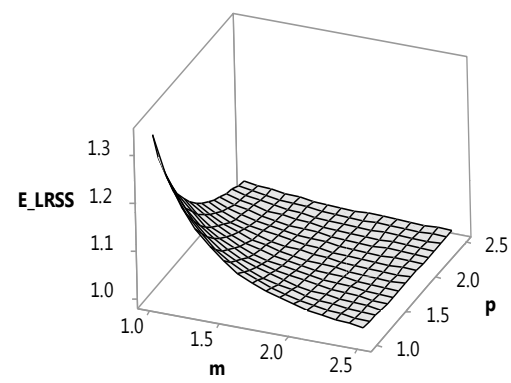

E_URSS with respec to $m$ and $p$ when $a=0.25$ and $n=10$

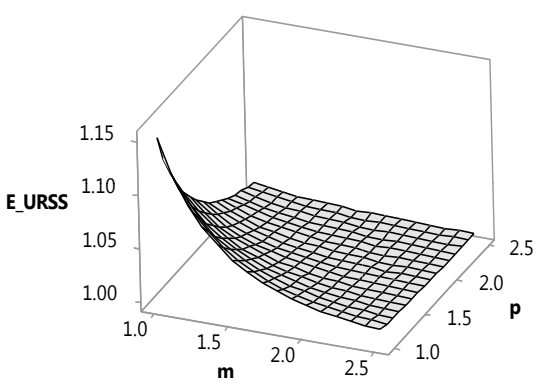

Observe that $\hat{\theta}_{2, L R S S}\left(\hat{\theta}_{2, U R S S}\right)$ is more efficient than $\hat{\theta}_{2, R S S}$ when $\alpha<0(\alpha>0)$. Efficiencies decreases up to 1 as $m$ and $p$ both increases or for fixed $m(p)$ with $p(m)$ increases and gets stabilize to one for larger $m$ and $p$. For larger values of $m$ and $p$, it is observed that $\hat{\theta}_{2, L R S S}\left(\hat{\theta}_{2, U R S S}\right)$ and $\hat{\theta}_{2, R S S}$ are equally efficient when $\alpha<0(\alpha>0)$.

Now, we summarize the overall conclusions about the efficiencies of estimators of $\theta_{2}$ based on various RSS schemes obtained in section 3 . 


\begin{tabular}{|c|c|c|c|}
\hline $\begin{array}{c}\widetilde{\theta}_{2} \\
\text { (Estimator } \\
\text { of } \boldsymbol{\theta}_{2} \text { ) }\end{array}$ & $\begin{array}{c}\widehat{\theta}_{2} \\
\text { Estimator } \\
\left.\text { of } \boldsymbol{\theta}_{2}\right) \\
\end{array}$ & $\begin{array}{c}\text { Efficiency } \\
\boldsymbol{e}\left(\widetilde{\boldsymbol{\theta}}_{2} \mid \widehat{\boldsymbol{\theta}}_{2}\right)=\frac{\operatorname{Var}\left(\widehat{\boldsymbol{\theta}}_{2}\right)}{\operatorname{Var}\left(\widetilde{\boldsymbol{\theta}}_{2}\right)}\end{array}$ & Remark about efficiency \\
\hline$\hat{\theta}_{2, R S S}$ & $\hat{\theta}_{2, S R S}$ & $e_{1}=\frac{n}{12 \sum_{r=1}^{n} \delta_{r}}$ & $\hat{\theta}_{2, R S S}$ is efficient than $\hat{\theta}_{2, S R S}$ \\
\hline$\hat{\theta}_{2, B L U E}$ & $\widehat{\theta}_{2, R S S}$ & $e_{2}=\frac{4}{n^{2}}\left(\sum_{r=1}^{n} \delta_{r}\right)\left(\sum_{r=1}^{n} \frac{\xi_{r}^{2}}{\delta_{r}}\right)$ & $\hat{\theta}_{2, B L U E}$ is efficient than $\hat{\theta}_{2, R S S}$ \\
\hline \multirow[b]{2}{*}{$\hat{\theta}_{2, L R S S}$} & $\hat{\theta}_{2, R S S}$ & $e_{3}=\frac{4 \xi_{1}^{2}\left(\sum_{r=1}^{n} \delta_{r}\right)}{n \delta_{1}}$ & \multirow{2}{*}{$\begin{array}{l}\hat{\theta}_{2, L R S S} \text { is efficient than } \hat{\theta}_{2, R S S} \text { and } \\
\hat{\theta}_{2, B L U E} \text { for } \alpha<0 \text { and } \hat{\theta}_{2, R S S} \\
\text { and } \hat{\theta}_{2, B L U E} \text { are efficient } \\
\text { than } \hat{\theta}_{2, L R S S} \text { for } \alpha>0 .\end{array}$} \\
\hline & $\hat{\theta}_{2, B L U E}$ & $e_{4}=\frac{n \xi_{1}^{2} / \delta_{1}}{\sum_{r=1}^{n} \xi_{r}^{2} / \delta_{r}}$ & \\
\hline \multirow{2}{*}{$\hat{\theta}_{2, U R S S}$} & $\hat{\theta}_{2, R S S}$ & $e_{5}=\frac{4 \xi_{n}^{2}\left(\sum_{r=1}^{n} \delta_{r}\right)}{n \delta_{n}}$ & \multirow{2}{*}{$\begin{array}{l}\hat{\theta}_{2, U R S S} \text { is efficient than } \hat{\theta}_{2, R S S} \text { and } \\
\hat{\theta}_{2, B L U E} \text { for } \alpha>0 \text { and } \hat{\theta}_{2, R S S} \\
\text { and } \hat{\theta}_{2, B L U E} \text { are efficient } \\
\text { than } \hat{\theta}_{2, U R S S} \text { for } \alpha<0 .\end{array}$} \\
\hline & $\hat{\theta}_{2, B L U E}$ & $e_{6}=\frac{n \xi_{n}^{2} / \delta_{n}}{\sum_{r=1}^{n} \xi_{r}^{2} / \delta_{r}}$ & \\
\hline \multirow{4}{*}{$\hat{\theta}_{2, E R S S_{1}}$} & $\hat{\theta}_{2, R S S}$ & $e_{7}=\frac{\sum_{r=1}^{n} \delta_{r}}{n \delta_{1}}$ & \multirow{8}{*}{$\begin{array}{l}\text { For } m=1 \text { and different } p \text {, and } \\
\hat{\theta}_{2, E R S S_{1}}, \hat{\theta}_{2, E R S S_{3}} \text { are more efficient } \\
\text { than } \hat{\theta}_{2, B L U E}, \hat{\theta}_{2, R S S} \text {. For } \alpha<0 \text {, } \\
\hat{\theta}_{2, L R S S} \text { is more efficient than } \\
\hat{\theta}_{2, E R S S_{1}}, \hat{\theta}_{2, E R S S_{3}}, \hat{\theta}_{2, U R S S} \text { and for } \\
\alpha>0, \hat{\theta}_{2, U R S S} \text { is more efficient } \\
\text { than } \hat{\theta}_{2, E R S S_{1}}, \hat{\theta}_{2, E R S S_{3}}, \hat{\theta}_{2, L R S S} \text {. }\end{array}$} \\
\hline & $\widehat{\theta}_{2, B L U E}$ & $e_{8}=\frac{n}{4 \delta_{1} \sum_{r=1}^{n} \xi_{r}^{2} / \delta_{r}}$ & \\
\hline & $\hat{\theta}_{2, L R S S}$ & $e_{9}=\frac{1}{4 \xi_{1}^{2}}$ & \\
\hline & $\hat{\theta}_{2, U R S S}$ & $e_{10}=\frac{1}{4 \xi_{n}^{2}}$ & \\
\hline \multirow{4}{*}{$\hat{\theta}_{2, E R S S_{3}}$} & $\hat{\theta}_{2, R S S}$ & $e_{11}=\frac{12 \sum_{r=1}^{n} \delta_{r}}{12(n-1) \delta_{1}+1}$ & \\
\hline & $\hat{\theta}_{2, B L U E}$ & $e_{12}=\frac{3 n^{2}}{\left(12(n-1) \delta_{1}+1\right)\left(\sum_{r=1}^{n} \xi_{r}^{2} / \delta_{r}\right)}$ & \\
\hline & $\hat{\theta}_{2, L R S S}$ & $e_{13}=\frac{3 n \delta_{1}}{\xi_{1}^{2}\left(12(n-1) \delta_{1}+1\right)}$ & \\
\hline & $\hat{\theta}_{2, U R S S}$ & $e_{13}=\frac{3 n \delta_{n}}{\xi_{n}^{2}\left(12(n-1) \delta_{n}+1\right)}$ & \\
\hline \multirow{2}{*}{$\hat{\theta}_{2, M E R S S}$} & $\hat{\theta}_{2, S R S}$ & $\frac{n}{24 \sum_{r=1}^{n / 2} \delta_{1}^{*}}$ & \multirow{2}{*}{$\begin{array}{l}\hat{\theta}_{2, M E R S S} \text { is efficient than } \hat{\theta}_{2, S R S} \cdot \\
\text { The performance of } \hat{\theta}_{2, M E R S S} \text { with } \\
\hat{\theta}_{2, R S S} \text { depends on parameter values } \\
\text { and } n \text {. }\end{array}$} \\
\hline & $\hat{\theta}_{2, R S S}$ & $\frac{\sum_{r=1}^{n} \delta_{r}}{2 \sum_{r=1}^{n / 2} \delta_{1}^{*}}$ & \\
\hline
\end{tabular}

\section{Conclusions}

In this paper, we consider a Morgenstern type bivariate generalized uniform distribution and obtain an unbiased estimator of scale parameter associated with a study variable based on different RSS schemes; usual RSS, LRSS, URSS, ERSS and MERSS. Further, BLUE of the scale parameter in case of censored RSS is also obtained. The efficiency performance of proposed estimators is also studied numerically and presented graphically. It is observed that, for $\alpha<0(\alpha>0)$ an estimator based on LRSS (URSS) is more efficient than that of estimator based on RSS, URSS (LRSS) and ERSS. 


\section{Acknowledgements}

The authors are grateful to the Reviewer and Editor of journal for the constructive comments which helped in the improvement of the paper.

\section{References}

[1] Al-Odat, M.T. and Al-Saleh, M.F. (2001). A variation of ranked set sampling. Journal of Applied Statistical Science 10(2), 137-146.

[2] Al-Saleh, M.F. and Al-Ananbeh, A.M. (2007). Estimation of the means of the bivariate normal using moving extreme ranked set sampling with concomitant variable. Statistical Papers 48(2), 179-195.

[3] Bain, L.J. (1978). Statistical analysis of reliability and life testing models: theory and methods. New York: Marcel Dekker.

[4] Bairamov, I., Kotz, S. and Bekci, M. (2001). New generalized Farlie-Gumbel-Morgenstern distributions and concomitants of order statistics. Journal of Applied Statistics 28(5), 521536.

[5] Bairamov, I. and Kotz, S. (2002). Dependence structure and symmetry of Huang-Kotz FGM distributions and their extensions. Metrika 56(1), 55-72.

[6] Barnett, V. and Moore, K. (1997). Best linear unbiased estimates in ranked-set sampling with particular reference to imperfect ordering. Journal of Applied Statistics 24(6), 697-710.

[7] Barnett, V. (1999). Ranked set sample design for environmental investigations. Environmental and Ecological Statistics 6(1), 59-74.

[8] Cambanis, S. (1977). Some properties and generalizations of multivariate Eyraud-GumbelMorgenstern distributions. Journal of Multivariate Analysis 7(4), 551-559.

[9] Chacko, M. and Yageen Thomas, P. (2007). Estimation of a parameter of bivariate Pareto distribution by ranked set sampling. Journal of Applied Statistics 34(6), 703-714.

[10] Chacko, M. and Thomas, P.Y. (2008). Estimation of a parameter of Morgenstern type bivariate exponential distribution by ranked set sampling. Annals of the Institute of Statistical Mathematics 60(2), 301-318.

[11] Chacko, M. (2016). Ordered extreme ranked set sampling and its application in parametric estimation. Journal of Statistical Theory and Applications 15(3), 248-258.

[12] Chen, Z., Bai, Z. and Sinha, B. (2004). Lecture Notes in Statistics, Ranked Set Sampling: Theory and Applications. New York: Springer

[13] David, H.A. and Nagaraja, H.N. (2003). Order Statistics. 3rd Edn. John Wiley and Sons, New York.

[14] Hanandeh, A.A. and Al-Saleh, M.F. (2013). Inference on Downton's bivariate exponential distribution based on moving extreme ranked set sampling. Austrian Journal of Statistics 42(3), 161-179.

[15] Huang, J.S. and Kotz, S. (1984). Correlation structure in iterated Farlie-Gumbel- 
Morgenstern distributions. Biometrika 71(3), 633-636.

[16] Huang, J.S. and Kotz, S. (1999). Modifications of the Farlie-Gumbel-Morgenstern distributions. A tough hill to climb. Metrika 49(2), 135-145.

[17] Koshti, R.D. and Kamalja, K.K. (2017). Estimation of scale parameter of a Bivariate Lomax distribution by Ranked Set Sampling. Model Assisted Statistics and Applications 12(2), 107113.

[18] Lam, K., Sinha, B. K. and Wu, Z. (1994). Estimation of parameters in a two-parameter exponential distribution using ranked set sample. Annals of the Institute of Statistical Mathematics 46(4), 723-736.

[19] Lam, K., Sinha, B.K. and Wu, Z. (1996). Estimation of location and scale parameters of a logistic distribution using a ranked set sample. Statistical Theory and Applications (pp. 187197). Springer, New York, NY.

[20] Lesitha, G. and Thomas, P. Y. (2013). Estimation of the scale parameter of a log-logistic distribution. Metrika 76(3), 427-448.

[21] McIntyre, G. A. (1952). A method for unbiased selective sampling, using ranked sets. Australian Journal of Agricultural Research 3(4), 385-390.

[22] Morgenstern D. (1956) Einfache Beispiele Zweidimensionaler Verteilungen. Mitteilingsblatt für Mathematische Statistik 8, 234-235

[23] Patil, G.P., Sinha, A.K. and Taillie, C. (1994). 5 Ranked set sampling. Handbook of statistics 12, 167-200.

[24] Samawi, H.M., Ahmed, M.S. and Abu-Dayyeh, W. (1996). Estimating the population mean using extreme ranked set sampling. Biometrical Journal 38(5), 577-586.

[25] Scaria, J., and Nair, N. U. (1999). On concomitants of order statistics from Morgenstern family. Biometrical Journal: Journal of Mathematical Methods in Biosciences 41(4), 483489.

[26] Scaria, J. and Thomas, B. (2008). Concomitants of order statistics from Generalized Morgenstern family. STARS 2(2), 153-162.

[27] Shaibu, A.B. and Muttlak, H.A. (2004). Estimating the parameters of the normal, exponential and gamma distributions using median and extreme ranked set samples. Statistica 64(1), 75-98.

[28] Singh, H.P. and Mehta, V. (2015). Estimation of scale parameter of a Morgenstern type bivariate uniform distribution using censored ranked set samples. Model Assisted Statistics and Applications 10(2), 139-153.

[29] Stokes, S.L. (1977). Ranked set sampling with concomitant variables. Communications in Statistics-Theory and Methods 6(12), 1207-1211.

[30] Stokes, S.L. (1980). Inferences on the correlation coefficient in bivariate normal populations from ranked set samples. Journal of the American Statistical Association 75(372), 989-995.

[31] Stokes, L. (1995). Parametric ranked set sampling. Annals of the Institute of Statistical Mathematics 47(3), 465-482. 
[32] Tahmasebi, S. and Jafari, A.A. (2012). Estimation of a scale parameter of Morgenstern type bivariate uniform distribution by ranked set sampling. Journal of Data Science 10(1), 129141.

[33] Tahmasebi, S. and Jafari, A.A. (2014). Estimators for the parameter mean of Morgenstern type bivariate generalized exponential distribution using ranked set sampling. Statistics and Operations Research Transactions 38(2), 161-180.

[34] Thomas, P. Y. and Veena, T. G. (2011). On an application of concomitants of order statistics in characterizing a family of bivariate distributions. Communications in Statistics-Theory and Methods 40(8), 1445-1452.

[35] Veena, T.G. and Thomas, P. Y. (2008). Characterizations of bivariate distributions by properties of concomitants of order statistics. Statistics \& Probability Letters 78(18), 33503354.

[36] Wolfe, D.A. (2004). Ranked set sampling: an approach to more efficient data collection. Statistical Science 19(4), 636-643. 